\title{
1 SIZE-DEPENDENT DIRECTED SOCIAL LEARNING IN NINE-SPINED
}

2 STICKLEBACKS

3

4 Grant A. Duffy, Thomas W. Pike ${ }^{\dagger}$ and Kevin N. Laland ${ }^{\S}$

5

6 School of Biology, University of St Andrews

7

$8{ }^{\S}$ Correspondence to Kevin N. Laland, School of Biology, St Andrews University, Bute Building,

9 Queen's terrace, St Andrews, Fife, KY16 9TS, UK.

10 Tel. 01334 463568, fax. 01334 463600, e-mail: knl1@st-andrews.ac.uk.

11

$12 \dagger$ Present address: Centre for Ecology and Conservation, University of Exeter, Penryn, UK 13

14 Running header: Copying in sticklebacks

15

16 Word Count: 4,588

17

18

19

20

21

22

23

24

25

26

27

28

29

30

31

32

33

34

35 
36 To forage efficiently in a patchy environment animals must make informed decisions concerning in

37 which patches to forage, for which the behaviour of other animals often provides informative cues.

38 However, other individuals may differ in the quality or relevance of information that they provide,

39 and accordingly animals are expected to be selective with respect to whom they copy. Such

40 selectivity may include the biasing of copying towards older, larger or more experienced

41 conspecifics. This study investigated whether the ability of nine-spined sticklebacks (Pungitius

42 pungitius) to exploit public information, that is, to judge the relative profitability of food patches

43 solely on the basis of the relative feeding activity of others, is influenced by their own body size and

44 that of the individuals from whom they copy. Individual observer fish, classed as either small or

45 large, were trained that two discrete foraging patches differed in their relative quality, one being

46 rich and the other poor ('personal information'). They then watched two shoals of either small or

47 large demonstrator conspecifics feeding at the two patches ('public information'), but with relative

48 profitability of the patches reversed compared to training, before being given the opportunity to

49 make a patch choice. Our results show that the effectiveness of this public demonstration is clearly

50 contingent on the size of the demonstrators, with subjects of both size classes copying the patch

51 choice of large demonstrators significantly more than they copied the patch choice of small

52 demonstrators. This study reinforces the view that animal social learning is directed along particular

53 pathways, with individuals predisposed by selection to copy particular categories of individual

54 differentially.

57 Keywords: foraging, nine-spined stickleback, patch assessment, public information, Pungitius

58 pungitius, social learning 
71 Efficient foraging in a patchy environment requires animals to make informed decisions concerning in which patches to forage and how long to spend at each patch. Relevant information capable of guiding such decision making can be obtained either directly, via sampling, or indirectly, by attending to social cues produced intentionally or inadvertently by other individuals (Giraldeau 1997; Kendal et al. 2005). Social learning, learning through observing others, reduces the costs associated with learning asocially, and potentially allows for faster location and resource estimation of patches, but can be costly if inappropriate or outdated information is acquired (Boyd \& Richerson 1985; Valone 2007).

The use of social learning may, however, be more complex than originally envisaged. Both evolutionary game theory and population genetic models lead to the prediction that animals ought to be highly selective with respect to the circumstances under which they rely on social learning and the individuals from whom they learn (Boyd \& Richerson 1985; Giraldeau et al. 2002). Animals should exhibit specific adaptive 'social learning strategies' that enhance the efficiency of asocial learning by selective or conditional use of both socially and asocially acquired information (Laland 2004). As a result, learned information may be directed along particular pathways, or between particular classes of individuals.

The differential transmission of acquired information along particular pathways was brought to prominence by Coussi-Korbel and Fragaszy (1995), who developed the concept of 'directed social learning'. Directed social learning refers to the idea that observing individuals evaluate the quality of information, and copy differentially, based on the identity of the demonstrator. Accordingly, individuals may be predisposed to copy successful, high status, or older individuals, or individuals in particular sex, age, or kinship classes (Coussi-Korbel and Fragaszy 1995). Previous studies provide evidence for directed social learning in relation to age (Choleris et al. 1997), sex (Katz and Lachlan 2003), familiarity (Swaney et al. 2001), and relatedness (Schwab et al. 2008).

Social learning is exhibited by a wide range of vertebrates, including many species of fish (Brown \& Laland, 2003), where the nine-spined stickleback, Pungitius pungitius, has proven a useful model system (Coolen et al. 2003, 2005; Van Bergen et al. 2004). Research into the use of social learning in patch quality evaluation has shown that while three-spined sticklebacks, Gasterosteus aculeatus, rely solely upon personal information and simple social cues, such as the number of conspecifics at a particular patch (Webster \& Hart 2006), nine-spined sticklebacks are able to use more complex social information, such as the feeding rate of other fish at a patch, in addition to these simpler ones (Coolen et al. 2003, 2005). The use of socially acquired information by nine-spined sticklebacks appears to be context specific, with individuals being more reliant on 
social information when personal information is unreliable, or is potentially outdated (Van Bergen et al. 2004).

There has hitherto been little research into directed social learning in sticklebacks, or even in fish in general. As of yet there is no indication that nine-spined sticklebacks are selective with respect to from whom they copy patch choices. To the contrary, nine-spines have been found to learn from social cues provided by heterospecifics, as well as conspecifics (Coolen et al. 2003). Nonetheless, there is a theoretical expectation that animals will preferentially learn from older individuals, since younger individuals may lack the experience to make effective judgments about patch and prey choice, which would leave copying them suboptimal (Laland 2004). Similarly, individuals may be predisposed to copy larger individuals, to the extent that size is indicative of factors such as long-term foraging success and greater age (i.e. increased survival). Consistent with this, Dugatkin and Godin (1993) reported age-dependent mate choice copying in guppies, with younger females acquiring mate preferences from older females (see also Amlacher and Dugatkin 2005).

This study examines the effect of demonstrator size on the use of socially acquired information concerning patch quality in both small and large nine-spined sticklebacks. We investigate whether the ability of these fish to exploit public information, that is, to judge the relative profitability of food patches solely on the basis of the relative feeding activity of others (Coolen et al. 2003), is size-dependent. "Observer" fish watch two shoals of "demonstrator" conspecifics feeding at different rates at two patches, and are then given the opportunity to make a patch choice, with the size of both observers and demonstrators manipulated. Large body size in sticklebacks may be due to either rapid growth, as a result of high foraging success (Wootton 1976), or increased age, since, like many fish species, sticklebacks exhibit continuous growth throughout life (Brown 1957). Large fish might thus be expected to be copied more than small fish, since their size is indicative of prior foraging success. Young observers are predicted to be more receptive to social cues than are older individuals, due to their relative naivety (Laland 2004), while older observers are thought generally less likely to use social information regardless of demonstrator age (Galef \& Whiskin 2004). For similar reasons, young demonstrators are predicted to be less effective transmitters of knowledge than older individuals. These predictions can be translated into expectations for the corresponding size classes.

\section{METHODS}

\section{(i) Collection and Holding of Fish}


Fish were collected, using dip nets, from Melton Brook, Leicester, UK, and transferred to 141 the aquarium the same day in plastic, water-filled containers. They were housed in either $30 \times 30$ $\mathrm{cm}$ or $30 \times 90 \mathrm{~cm}$ tanks (water level $18 \mathrm{~cm}$ ) in groups of up to 15 or $45 \mathrm{fish}$, respectively. The first batch of fish was collected in November 2006, while the second batch was collected during

144 November 2007. Both batches were approximately the same average size and at the same stage of development at the time of capture. However, the 2006 batch was reared in captivity for one year longer so that at the time the experiment took place (between February and September 2008) the batches formed two discrete groups comprised of large (2006) and small (2007) fish. Large fish had a standard length greater than $40 \mathrm{~mm}$ (mean $\pm \mathrm{SE}$ from a random sample of $N=30$ individuals: $45.7 \pm 0.63 \mathrm{~mm}$ ) and small fish were less than $35 \mathrm{~mm}$ (mean $\pm \mathrm{SE}: 31.0 \pm 0.43 \mathrm{~mm}, N=30)$ at the start of the experiment, and there was a significant difference between the two groups $\left(t_{58}=18.53\right.$, $P<0.001)$. Fish in both groups grew throughout the period of the experiment, although on completion there was still a significant size difference between them (large fish, mean \pm SE: $47.3 \pm$ $0.61, N=30$; small fish, mean $\left.\pm \mathrm{SE}: 34.1 \pm 0.68, N=30 ; t_{58}=14.38, P<0.001\right)$. Although fish appeared, from visual observation, to have been born the year of capture, this was not definitely known. Fish were kept in several separate holding tanks, categorised by size and whether they would be used as focal or demonstrator fish in the experiments (see below), in a cold room with an ambient temperature of $7-9{ }^{\circ} \mathrm{C}$ and water temperature of $8-9{ }^{\circ} \mathrm{C}$. The cold room was kept on a stable 12L: 12D light-cycle in order to reduce potential interference from reproductive behaviour, which has been found to affect fish behaviour (Pitcher 1996). Fish were fed daily on a diet of frozen bloodworms. Focal and demonstrator fish were reared in separate holding tanks to prevent any familiarity developing between them. It is highly unlikely that any familiarity developed in the wild, over 4 months (small fish) or 16 months (large fish) before the experiment, would still be

(ii) Experimental Setup

The experimental tank $(45 \times 30 \times 30 \mathrm{~cm}$; water level $17 \mathrm{~cm})$ was divided into three sections using removable transparent partitions (Fig. 1). Two feeding columns (30 cm high), one coloured yellow and one blue, were located in the centre of one of the sides of the tank. The transparent fronts of the feeders were visible only to fish within the 'goal zones', while only the opaque sides were visible from the observer compartment, which was located on the opposite side of the tank (Fig. 1). The tank was blacked-out on all sides to prevent any external stimuli, such as movements made by the experimenter, affecting fish behaviour. A video camera positioned $50 \mathrm{~cm}$ above the tank and connected to a laptop computer provided a plan view of the tank and digitally recorded all experimental proceedings. 
A total of 120 sticklebacks were divided equally into 2 groups, one consisting of large individuals and the other small fish randomly selected from the stock populations, and the experiment conducted as follows:

\section{(a) Personal-information training}

Fish were trained to become accustomed to the feeding columns, and to acquire personal information about which feeder provided most food (the 'rich' patch) and which the least (the 'poor' patch). Sticklebacks have been previously shown to associate patch richness with the colour and position of a specific feeder (Girvan \& Braithwaite 1998). Training occurred in groups of 10 fish of the same size class, during which fish were placed into the experimental tank and confined within either the rich or poor zone using transparent Perspex barriers. Fish were allowed to acclimatise for $5 \mathrm{~min}$ before a $10 \mathrm{~min}$ feeding period began. The feeding regime for the rich feeder was one bloodworm every $90 \mathrm{~s}$ for the entire $10 \mathrm{~min}$, while the regime for the poor patch was one bloodworm after the initial $90 \mathrm{~s}$ and one $270 \mathrm{~s}$ into the $10 \mathrm{~min}$ period. A small amount of water in which bloodworms had been defrosted was added every $90 \mathrm{~s}$ when following a poor feeding regime to ensure visual rather than olfactory cues were used by focal fish in determining patch quality during the final experimental stage. We have established that subjects exposed to this procedure, but denied visual access to demonstrators, when tested chose the zone formerly housing the richer and poorer patches at random (Van Bergen 2004), demonstrating that our procedures successfully mask any residual olfactory cues. Every subgroup underwent two training periods, one rich and one poor, every day for four days, a total of eight training periods for each group. Preliminary experimentation showed this to be sufficient to alter patch preferences from the expected patch choice if choices occurred purely at random. The first feeding of each day alternated between rich and poor patches in order to ensure maximum fairness in patch evaluation resulting from this training. In order to reduce the effect of bias for either colour or side of tank, each experimental group was counterbalanced such that the rich patch feeder was equally frequently the blue or yellow feeder, and on the left or right side of the tank.

\section{(b) Demonstration}

For the demonstration stage, a third of the small and a third of the large focal fish experienced a public demonstration from large demonstrators, a third from small demonstrators and the remaining third, the controls, saw no demonstration but experienced a time delay of equivalent duration. A single focal fish was placed inside the observer compartment within the experimental tank, with an opaque removable barrier preventing the focal fish from seeing the rest of the tank. Into each of the goal zones the experimenter placed 3 large, 3 small, or no fish, depending on the 
210 experimental or control group. Demonstrators were confined to their zone by transparent Perspex

211 barriers (Fig. 1). All fish were then left for a period of 5 min to acclimatise in the experimental tank. After the acclimatisation period, the opaque partition obscuring the view of the tank from the observer compartment was removed. The same feeding regimes as utilised during training were

214 deployed for the two feeders, with one feeder following the rich regime and the other following the poor regime. The configuration of these was directly opposite to the one employed for training, so

216 that the personal information possessed by the fish conflicted with the social cues provided by the 217 demonstrators. For example, if the focal fish was trained with the rich patch being provided by the

218 blue feeder on the left side of the tank, then for demonstration the yellow feeder on the right side of the tank would provide the richer patch. Following a 10 min demonstration period the opaque divider was once again placed in the tank to restrict the view from the observer compartment. All demonstrator fish were returned to holding tanks, and both transparent dividers were removed from the experimental tank in preparation for the final testing stage. Any remaining bloodworms in the tank were also removed to ensure that the only cues available to focal fish were social ones. Following the eighth and final training trial, focal fish were not fed for 24 hours to ensure sufficient motivation to induce foraging behaviour on the fifth day, when fish were tested for a foraging patch

\section{(c) Behavioural testing}

The behavioural test stage of the procedure began with the removal of the observer compartment, releasing the focal fish into the experimental tank. The behaviour of the focal fish was monitored and recorded, via the laptop computer, for $90 \mathrm{~s}$ after its release into the experimental tank. Pilot work established that the response to social cues is most prevalently seen during this initial $90 \mathrm{~s}$ period. The first goal zone into which a fish entered was noted, along with the total amount of time the fish spent within the rich goal zone, and these used as variables in the analysis. A fish was designated within a goal-zone when the front of its body, up to its pectoral fins, was within the zone. Goal-zone preference is described in terms of 'public-rich' (or 'personal-poor'; i.e. the foraging patch that fish learned was poor during personal training, but later demonstrated to be rich) and 'public-poor' (or 'personal-rich'). 
Differences between observer and demonstrator sizes classes on the first goal-zone entered were compared using a Generalized Linear Model (GLM) with a binomial error structure and a logit link function. Similar comparisons were made for the time spent in the rich goal zone, using a GLM with negative binomial errors. $N$ refers to the sample size (number of fish). All tests are two-tailed.

(iv) Ethical Note

No fish died during the study. After the trials the fish were retained in the laboratory, some of which may be used as breeding stock, until they die of natural causes (lifespan in captivity ca.1-2 years). Nine-spined sticklebacks are extremely common at the location from which they were collected, and the removal of individuals for use in this study is unlikely to have had any negative ecological consequences. No licence was required for the study, and the fish were not subjected to any pain or distress. The fishes' condition was continuously monitored by a dedicated Named Animal Care and Welfare Officer (NACWO), who ensured they were kept in a suitable environment and were in good health, and they are subject to monthly visits from the Home Office

\section{RESULTS} Inspector and the University's veterinarian.

In the control groups, which did not see public demonstrations, both small and large focal fish showed a significant preference for the personal-rich patch (i.e. the patch that they learned was rich during personal training). Fewer fish entered the public-rich goal zone first than expected by chance (binomial tests against an expected proportion of 0.5, small fish: $P=0.019$; large fish: $P=$ 0.019 ) and individuals spent significantly more time in the personal-rich over the personal-poor foraging patch (Mann-Whitney tests, small fish: $U=306.0, N_{1}=N_{2}=20, P=0.005$; large fish: $U=$ 282.0, $N_{1}=N_{2}=20, P=0.001$; Fig. 2), confirming that the personal-information training was successful.

While the time spent in the public-rich rich feeding patch did not differ between large and small observers (GLM: $z=0.18, P=0.86$ ), it was significantly affected by the demonstrators' size class $(z=3.01, P=0.003)$, such that both small and large observer individuals spent significantly more time in the public-rich zone following a demonstration by large fish than after a demonstration by small fish (small observers: $z=3.04, P=0.002$; large observers: $z=6.22, P<0.001$ ) (Fig. 2b). The proportion of fish entering the public-rich goal zone first exhibited a trend in the same direction. This proportion was only weakly affected by the size class of the demonstrators (GLM: $z$ $=1.57, P=0.12)$ and did not differ between large and small observers $(z=0.68, P=0.49)$, although 
there was a trend towards a preference for foraging patches demonstrated by large demonstrators

281 (especially for large observers) (Fig. 2a).

\section{DISCUSSION}

The findings of this study imply that nine-spined sticklebacks practice directed social learning, utilizing social cues from conspecific demonstrators depending upon the size or age of the demonstrator, consistent with their deployment of either a 'copy successful individuals' or 'copy larger (potentially older) individuals' social learning strategy (Laland 2004).

The behaviour of the fish in the control conditions, who did not receive public information, illustrates that the personal training was effective, since these fish exhibited a strong preference for the personal-rich foraging patch at test. Nonetheless, for many of fish in the experimental conditions, one 10 min feeding demonstration by conspecifics proved sufficient to alter their patch choice. However, the effectiveness of this demonstration is clearly contingent on the size of the demonstrators, with subjects of both size classes copying the patch choice of large demonstrators significantly more than they copied the patch choice of small demonstrators. These findings suggest that the sticklebacks are discriminating between social cues based upon their source, consistent with the hypothesis that the behaviour of small focal fish would be more easily influenced by larger conspecifics than by smaller ones (Laland 2004). An alternative interpretation is that the observation of demonstrators behaving differently to the subjects' prior behaviour merely undermines the subjects' preference for the personal-rich patch, leading to random behaviour at test in the experimental groups. However, this alternative account is inconsistent with the findings of previous studies using the same procedures (van Bergen et al., 2004; Kendal et al., 2009), which reveal that the magnitude by which subjects select the public-rich patch can be incremented by increasing the returns to demonstrators or the noisiness of personal training, and decreased by the reverse manipulations, to the point where strong patch preferences can be demonstrated. Such manipulations imply that the public demonstration does more than merely erode prior personal experience, and induces learning. In this study the levels of personal training and public demonstration were carefully selected so as to minimize the chances that ceiling or floor effects would hide differences between experimental conditions.

It is tempting to interpret these findings as indicating that nine-spined sticklebacks possess specialized evolved psychological mechanisms predisposing them to size-dependent directed social learning, and leading to their utilizing social information stemming from large conspecifics more frequently than that from small demonstrators. However, we cannot rule out the alternative 
315 hypothesis that the observed directed social learning results because large fish produce more

316 conspicuous or coherent social cues than small fish. While we also cannot rule out the possibility

317 that our results were influenced by differences between batches in the year they were captured or

318 the time subsequently spent in the lab, we consider this explanation unlikely. In other experiments

319 conducted in our laboratory on individuals collected in different years, or held for differing periods

320 of time, nine-spined sticklebacks have behaved consistently (Coolen et al., 2003, 2005; van Bergen

321 et al. 2004; Kendal et al., 2009)

322 Also of interest is the observation that large focal fish appear to be even more receptive than

323 small fish to cues from large demonstrators. The results of this study suggest than larger individuals

324 will use socially-acquired information over personal information when demonstrators are of a

325 similar size and age, and do so to a greater extent than smaller and younger observers. This is likely

326 to have direct benefits, as spending more time in a rich patch and less in a poor patch will reap

327 foraging dividends, but appears to contradict Galef and Whiskin's (2004) suggestion that older

328 individuals should be less likely to use socially gained information regardless of source. One

329 explanation is that due to ontogenetic shifts in foraging niche, the types (and specifically sizes) of

330 food exploited by large fish may differ substantially from those preferred by small fish (Wootton

331 1976). Foraging activity by small fish may thus provide large individuals with accurate information

332 regarding the presence of food but be a poor indicator of the presence of preferred food types,

333 explaining their greater tendency to copy larger conspecifics.

334 The experience that typically comes with age may be the reason why small (and large)

335 sticklebacks appear to value social information from large demonstrators above that of small ones.

336 An older fish has passed a selective filter, in the sense that its behaviour has been successful enough

337 to keep it alive thus far. By such reasoning, suboptimal behaviour might be expected to reach a

338 higher frequency in younger than older individuals, and to be increasingly weeded out by selection

339 as individuals age. If this is correct, differentially copying from older individuals should be

340 adaptive, and size is a reliable cue of age in sticklebacks (Wootton 1976). Alternatively, large

341 demonstrators may be preferentially copied directly for their size. For instance, larger individuals

342 may be perceived to be more successful than smaller ones (Candolin \& Voigt 2001), with

343 individuals pursuing a 'copy the successful' strategy. The close correlation between age and size in

344 fish (Brown 1957) leaves disassociating these hypotheses extremely challenging. However, recent

345 work within our laboratory indicates that nine-spined sticklebacks are more inclined to copy

346 successful than unsuccessful size- and age-matched demonstrators. Irrespective of the precise

347 strategy being pursued, the study provides strong support for the arguments that social learning is

348 not random, but directed (Coussi-Korbel \& Fragaszy 1995), and that animals rely on evolved social

349 learning strategies that dictate from whom they learn (Laland 2004). 
Acknowledgements

353

We thank two anonymous referees for comments, which greatly improved the manuscript. This research was supported in part by a BBSRC grant to KNL (BB/C005430/1).

\section{References}

Amlacher, J. \& Dugatkin, L. 2005. Preference for older over younger models during mate-choice copying in young guppies. Ethology Ecology \& Evolution, 17(2), 161-169.

Boyd, R. \& Richerson, P. J. 1988. An evolutionary model of social learning: the effects of spatial and temporal variation. In Social learning: psychological and biological perspectives (Ed by P. R. Zentall \& B. G. Galef Jr), pp. 29-48. Hillsdale, NJ: Lawrence Erlbaum Associates.

Brown, C. \& Laland, K. 2003. Social learning in fishes: A review. Fish and Fisheries, 4(3), 280288.

Brown, M.E. 1957. The Physiology of Fishes. New York: Academic Press Inc.

Candolin, L. \& Voigt, H. 2001. Correlation between male size and territory quality: Consequence

Choleris, E., Guo, C., Liu, H.F., Mainardi, M. \& Valsecchi, P. 1997. The effect of demonstrator age and number on duration of socially-induced food preferences in house mouse (Mus of male competition or predation susceptibility. Oikos, 95(2), 225-230. domesticus). Behavioural Processes, 41(1), 69-77. rely on public information over simpler social cues. Behavioural Ecology, 16(5), 865-870. 
386 Coussi-Korbel, S. \& Fragaszy, D. 1995. On the relation between social dynamics and social learning. Animal Behaviour, 50(6), 1441-1453. doi: 10.1016/0003-3472(95)80001-8

Dugatkin L.A. \& Godin J.G. 1993. Sexual selection and female copying: age dependent effects. Behavioural Ecology 4, 289-292.

Galef, B.G. \& Whiskin, E. 2004 Effects of environmental stability and demonstrator age on social learning of food preferences by young norway rats. Animal Behaviour, 68, 897-902. doi:

Giraldeau, L. 1997. The ecology of information. In Behavioural ecology: An evolutionary approach, 4th edition (Ed by Krebs, J. \& Davies, N.). pp. 42-68. Oxford: Blackwell Scientific.

Giraldeau, L.A., Valone, T. J. \& Templeton, J. J. 2002. Potential disadvantages of using socially acquired information. Philosophical Transactions of the Royal Society of London B-Biological

Girvan, J. \& Braithwaite, V. 1998. Population differences in spatial learning in three-spined sticklebacks. Proceedings of the Royal Society of London Series B-Biological Sciences, 265(1399), 913-918.

Katz, M. \& Lachlan, R. 2003. Social learning of food types in zebra finches (Taenopygia guttata) 409

Kendal, JR., Rendell L., Pike TW \& Laland KN 2009 Nine-spined sticklebacks deploy a hillclimbing social learning strategy. Behavioural Ecology doi:10.1093/beheco/arp016

Kendal, R.L., Coolen, I., Van Bergen, Y.\& Laland, K.N. 2005. Tradeoffs in the adaptive use of

Laland, K. 2004. Social learning strategies. Learning \& Behaviour, 32(1), 4-14. social and asocial learning. Advances in the Study of Behaviour, 35, 333-379. 
420 Schwab, C., Bugnyar, T., Schloegl, C. \& Kotrschal, K. 2008. Enhanced social learning between

421 siblings in common ravens, Corvus corax. Animal Behaviour, 75, 501-508. doi:

424 Swaney, W., Kendal, J., Capon, H., Brown, C. \& Laland, K. 2001. Familiarity facilitates social 425 learning of foraging behaviour in the guppy. Animal Behaviour, 62, 591-598. doi:

426 10.1006/anbe.2001.1788

Utne-Palm, A.C. \& Hart, P.J.B. 2000. The effects of familiarity on competitive interactions between three-spined sticklebacks. Oikos, 91. 225-232.

Valone, T.J. 2007. From eavesdropping on performance to copying the behavior of others: A review of public information use. Behavioural Ecology and Sociobiology, 62, 1-14.

Van Bergen, Y. 2004. Unpublished Ph.D. Thesis, University of Cambridge.

Van Bergen, Y., Coolen, I. \& Laland, K. 2004. Nine-spined sticklebacks exploit the most reliable source when public and private information conflict. Proceedings of the Royal Society of London Series B-Biological Sciences, 271(1542), 957-962. doi: 10.1098/rspb.2004.2684

Webster, M. \& Hart, P. 2006. Subhabitat selection by foraging threespine stickleback

(Gasterosteus aculeatus): Previous experience and social conformity. Behavioural Ecology and Sociobiology, 60(1), 77-86.

Wootton, R.J. 1976. The biology of the sticklebacks. London: Academic Press. 
471 Figure 1. Diagram of the experimental tank, as used during demonstration periods. Solid black lines 472 represent opaque surfaces, dotted lines represent transparent surfaces, and dashed lines represent 473 removable transparent dividers and delimit the goal-zones used during the test phase.

475 Figure 2. (a) Proportion of small and large focal fish within each group entering the public-rich 476 (personal-poor) goal-zone first during the test period ( $N=20$ for each group). (b) Median \pm 477 interquartile range and maximum and minimum values of time that focal fish from each group spent 478 within the public-rich goal-zone during the $90 \mathrm{~s}$ test period. Fish in control groups saw no public 479 demonstration. Fish in experimental groups saw a demonstration by either large or small 480 demonstrators (see text for full details). 


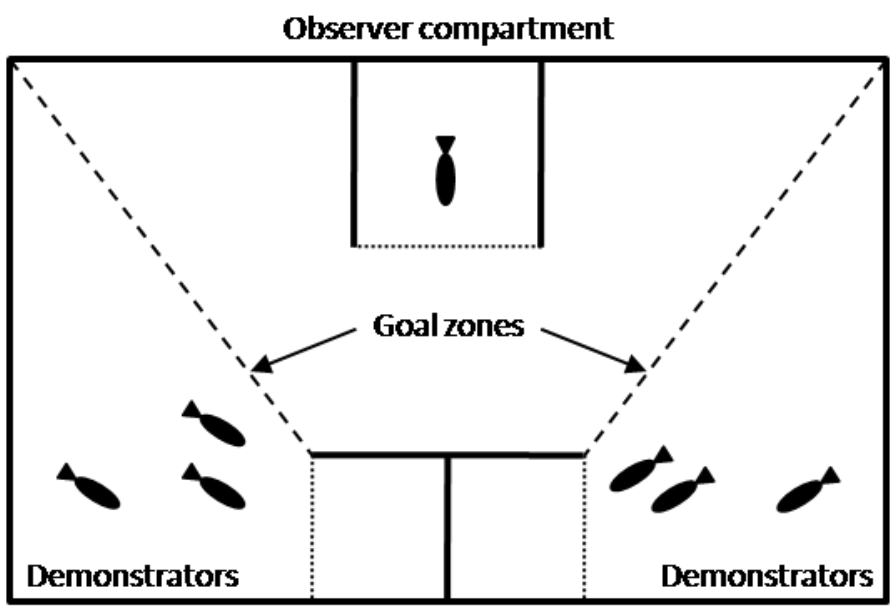

503

Feeders

504 Duffy et al., Figure 1

505

506

507

508

509

510

511

512

513

514

515 

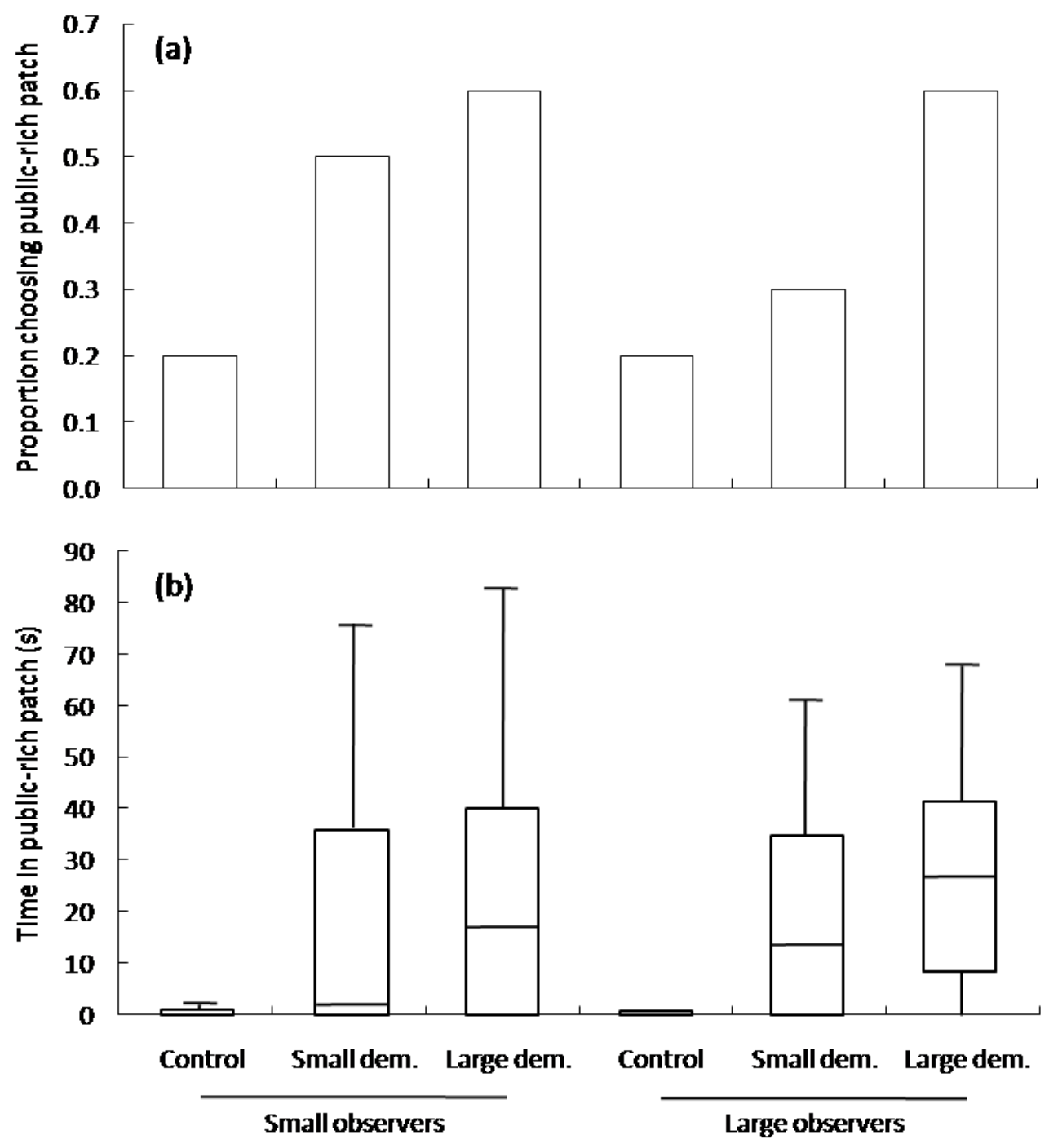

Duffy et al., Figure 2 